\title{
Contents, Vol. 94, 1938
}

\section{Inhaltsverzeichnis.}

Eigenarbeiten. seite

Böck, J. und Schlagenhauff, K., Ueber das Vorkommen

von Onkozyten in der menschlichen ТГӓnendrüse . 244

Csapody, von Istvan, Neuere Erfahrungen mit der Höhlen-

plastik 23

Davids, H., Zur Frage der Behandlung der Tuberkulose

des Auges 305

Fazakas, A., Ueber die Bedeutung der Pilze für die Augen-

heilkunde 253

Fischer, Franz, Die Fehlbildung der ableitenden Tränen-

wege und ihre formale Genese 1, 152

Hoehne, H., Ueber das Auftreten reflektorischer Zweck-

bewegungen zur Verbesserung des Sehaktes beim

Säugling 241

Jakobowits, R., Strangbildung in der Vorderkammer nach

Keratitis parenchymatosa 325

Meisner, W., Zur Behandlung der Keratoconjunctivitis sicca 129 Purtscher, Ernst, Zur Aetiologie der «spontanen» Aderhaut-

abhebung und der «serösen Tenonitis» . . 12, 141 Rintelen, F., Zur Differentialdiagnose der

Aderhautsarkome 320 Scheyhing, H., Isolierte Lähmung des Naheinstellungszen-

trums nach Schädelbasisbruch 315

Schlagenhauff, K., siehe Böck, J.

Schutzbach, M., Ueber einen Fall von schwerer Mißbildung,

insbesondere des Schädels und des Gesichtsskeletls . 131

Aus der Praxis für die Praxis.

Schweig, S. J., Ueber Salbenfüllung des Bindehautsackes . 162 Weckert, Fusionszentrum und

Schielen .... . 258

IV

Inhaltsverzeichnis

Berichte über die ophthalmologische Literatur. Seite

Entwicklungsgeschichte des Auges, Mißbildungen, Anthro-pologie. (Berichtszeitraunt: 1. Juli

1936 bis 30. Juni1937). Von R. Seefelder-Innsbruck 34

Geschichte de $\Gamma$ Augenheilkunde. (Berichtszeitraum: 1. Juli

1936 bis 30. Juni 1937.) Von R. Seef elder-Innsbruck . 56

Normale und pathologische Anatomie. Von Prof. Dr. Rudolf Schneíder-GrAz

Aderhaut, Netzhaut. (Berichtsjahr vom 1. Januar bis 31. De-

zember 1936.) Von Prof. Dr. W. Ríehm-Gießen 78, 164 
Physiologische und psychologische Optik. (Berichtsjahrvom 1. Oktober 1936 bis 30. September 1937.) VonDr. Rudolf Sf ieòer-Innsbruck . . . . 202

Vererbung und Auge. (Berichtsjahr 1936-1937.) Von A.

Franeeschetti-Genf 266, 329

Ophthalmologische Therapie:I. Medikamentöse Therapie. (Berichtsjahr vom 1. Oktober 1936 bis 30. September 1937.) Von E. Kunz-Bẹ́m 342II. Operative Therapie. (Berichtsjahr vom 1.

Oktober 1936bis 30. September 1937.) Von W. Hoffmann-Königs-berg i. Pr 349

III. Physikalische und Strahlentherapie. Von W. Hoff-

marm-Königsberg i. Pr 359

Gesellschaftsberichte.

Ophthalmologische Gesellschaft in Wien:

Sitzung vom 18. Oktober 1937

295

Sitzung vom 22. November 1937

298

Sitzung vom 17. Januar 1938 366

2.T. Tagung märkischer Augenärzte in Berlin am 12. und 13. Juni 1937 98Wissenschaftliche Sitzung der augenärztlichen Vereinigung des Ober- 